\title{
Multi-wavelength Study of the Be/X-ray Binaries
}

\author{
J. Z. Yan, H. Li, J. N. Zhou, and Q. Z. Liu ${ }^{1,2, *}$ \\ ${ }^{1}$ Puple Mountain Observatory, Chinese Academy of Sciences, Nanjing 210008, China \\ ${ }^{2}$ Key Laboratory of Dark Matter and Space Astronomy, Chinese Academy of Sciences, \\ Nanjing 21008, China \\ *email: jzyan@pmo.ac.cn
}

\begin{abstract}
An anti-correlation between the optical brightness and the strength of the $\mathrm{H} \alpha$ emission was observed from two Be/X-ray binaries, A0535+26 and MXB 0656-072: when the optical brightness showed a decrease, the intensity of the $\mathrm{H} \alpha$ emission displayed an obvious increase. This anti-correlation is interpreted as the result of the mass ejection from the Be star. After the mass ejection event, a cavity or low-density region will be developing in the inner parter of the circumstellar disk. The X-ray outbursts of the Be/X-ray binaries might be connected with the mass ejections from the Be star.
\end{abstract}

Keywords. Stars: neutron, stars: emission line, X-rays: binaries.

\section{Introduction}

$\mathrm{Be} / \mathrm{X}$-ray binary represents a major subclass of high mass X-ray binary in our Galaxy (Liu et al. (2006)). A neutron star moves in a wide and eccentric orbit around the Be star, which is a non-supergiant rapid-rotating B-type star and ever shows Balmer emission lines at least once in its life (Porter \& Rivinius (2003)). There are two different disks in Be/X-ray binary: a circumstellar disk around Be star and an accretion disk around neutron star (see Reig (2011)) and the reference therein).

The optical thick $\mathrm{H} \alpha$ emission line in Be star is generally believed to be formed in the entire circumstellar disk (Slettebak et al. (1992)), while only the innermost part of the disk contributes significantly to the continuum flux. Due to the higher ionization potential energy, the formation region of the HeI $\lambda 6678$ line should be smaller than the nearby continuum region (Stee et al. (1998)). The increase of the $\mathrm{H} \alpha$ intensity indicates that an extended circumstellar disk is forming around Be star, while the decrease of the V-band brightness suggests that a low-density or cavitated region is developing in the inner part of the disk.

\section{The Anti-correlation between the $\mathbf{H} \alpha$ Intensity and the $V$-band Brightness}

We carried out the optical spectroscopic observations on two Be/X-ray binaries, A0535+26 and MXB 0656-072, with the 2.16m telescope at Xinglong Station, NAOC. The anti-correlation between the $\mathrm{H} \alpha$ emission and the $V$-band brightness, a low V-band emission level with a strong $\mathrm{H} \alpha$ emission, was observed from both $\mathrm{Be} / \mathrm{X}$-ray binaries.

We observed a strong $\mathrm{H} \alpha$ emission, with an EW of $-25 \AA$, in the Be/X-ray binaries A0535+26, during our 2009 observations, which was carried out just before the 2009 strong X-ray outburst of the system. In the same time, the $V$-band brightness of the system showed an obvious decrease (see Fig. 4 in Yan et al. (2012a)). The increase of 
the $\mathrm{H} \alpha$ emission might be caused by the decrease of the continuum emission. In fact, the $\mathrm{H} \alpha$ emission of $\mathrm{A} 0535+26$ was in a low level while the $V$-band brightness was still in a decline phase during our 2010 observations (see Fig. 4 in Yan et al. (2012a)). Therefore, we suggest the anti-correlation between the $\mathrm{H} \alpha$ emission and the $V$-band brightness during our 2009 observations should be caused by the physical changes in the circumstellar disk around Be star. The same phenomenon was also observed in the Be/X-ray binary, MXB 0656-072, during our 2007 observations, which was taken just before a Type I X-ray outburst of the system (see Fig. 8 in Yan et al. (2012b)).

\section{Mass ejections from the Be Star}

The similar observational results were also observed in other Be/X-ray binaries, such as 4U 1145-619 (Stevens et al. (1997)) and 4U 0115+63 (Reig et al. (2007)). It should be a common feature in the Be/X-ray binaries. Observational results (Rivinius et al. (2001)) and theoretical calculations (Meilland et al. (2006)) suggest that a low-density region seems to develop around the Be star after an outburst. It was suggested that the outburst might be connected with the increased mass loss or mass ejection from the Be star. Some weeks to months after the outburst, the stellar radiation pressure could gradually excavates the inner part of the disk and a low-density region would develop around the Be star and slowly grow outward. With the vacuation of the inner disk, the optical continuum emission decreases and an increase in $U B V$ magnitudes will be observed. After the outburst, material is transferred into the disk and a more extended circumstellar disk should be formed, which produces the stronger $\mathrm{H} \alpha$ emission from the system. The new disk material might be ejected from the star by the subsequent mass ejection or reaccreted into the inner region after the supply of material from the star to the disk has been turned off (Clark et al. (2001)).

\section{Conclusions}

The anti-correlation between the optical brightness and the intensity of the $\mathrm{H} \alpha$ emission in the Be/X-ray binaries could be explained by the mass ejections from the Be star. The mass ejection could also trigger the following X-ray outbursts.

This work was partially supported by the National Basic Research Program of China - 973 Program 2009CB824800, and the National Natural Science Foundation of China under grants 11003045 .

\section{References}

Cark et al., 2001, A\&SA, 380, 615

Liu et al., 2006, A\& $A, 455,1165$

Porter, J. M. \& Rivinius, T., 2003, PASP, 115, 1153

Reig, P., 2011, Astrophysics and Space Science, 332, 1

Reig, P., Larionov, V., Negueruela, I., et al., 2007, A\& A, 462, 1081

Slettebak et al., 1992, ApJS, 81, 335

Stee et al., 1998, A\&BA, 332, 268

Stevens, J. B., Reig, P., Coe, M. J., et al., 1997, MNRAS, 288, 988

Rivinius et al., 2001, A\&A, 379, 257

Meilland et al., 2006, A\&A, 455, 953

Yan, J. Z., Li, H., \& Liu, Q. Z., 2012, ApJ, 744, 37

Yan, J. Z., Chaty, S., Zurita Heras, J. A., Li, H., \& Liu, Q. Z., 2012b, ApJ, 753, 73 